\title{
WDM compatible and electrically tunable SPE-OCDMA system based on the temporal self-imaging effect
}

\author{
S. Tainta ${ }^{1, *}$ W. Amaya, ${ }^{2}$ M. J. Erro, ${ }^{1}$ M. J. Garde,${ }^{1}$ S. Sales, ${ }^{2}$ and M. A. Muriel ${ }^{3}$ \\ ${ }^{1}$ Departamento de Ingeniería Eléctrica y Electrónica, Universidad Pública de Navarra, Pamplona, 31006, Spain \\ ${ }^{2}$ ITEAM Research Institute, Universidad Politécnica de Valencia, Valencia, 46022, Spain \\ ${ }^{3}$ Tecnología Fotónica, ETSIT, Universidad Politécnica de Madrid, Madrid, 28040, Spain \\ *Corresponding author: santiago.tainta@unavarra.es
}

Received October 29, 2010; revised December 23, 2010; accepted December 27, 2010; posted January 7, 2011 (Doc. ID 137378); published January 31, 2011

A coding/decoding setup for a spectral phase encoding optical code-division multiple access (SPE-OCDMA) system has been developed. The proposal is based on the temporal self-imaging effect and the use of an easily tunable electrooptic phase modulator to achieve line-by-line coding of the transmitted signal, thus assuring compatibility with WDM techniques. Modulation of the code is performed at the same rate as the data, avoiding the use of high-bandwidth electro-optic modulators. As proof of concept of the technique, experimental results are presented for a back-to-back coder/decoder setup transmitting a $10 \mathrm{GHz}$ unmodulated optical pulse train within an $80 \mathrm{GHz}$ optical window and using 8-chip Hadamard codes. (c) 2011 Optical Society of America

OCIS codes: $\quad 060.0060,060.5060,060.4230,060.3735,320.5540$.

Optical code-division multiple access (OCDMA) [1] has generated an increasing interest for applications in highbandwidth access networks. Among many different approaches, spectral-phase-encoded OCDMA (SPEOCDMA) [2] has gained special attention through its successful application as an efficient communication system in metropolitan access networks [3]. This technique is based on the assignment of a predetermined group of phase shifts to the different frequency bins of an ultrashort optical pulse. A different code that specifies the phase shifts is assigned to each user, requiring the application of the complementary code at the receiver to recover the original information. Several proposals have been made based on this technique using different technologies, such as volume optics [2], arrayed waveguide gratings [3] , or fiber-dispersive elements [4, 5$]$ ].

Another major goal in OCDMA systems is to attain spectral efficiency by reducing the bandwidth transmitted to a certain spectral window and thus guarantee compatibility with WDM systems. To achieve this, reduction of the bandwidth occupied by the transmitted signal is mandatory, and it can be obtained by reducing the spectral width of the different frequency bins so that each one contains only one spectral line. This way, each chip of the code word is associated to an individual spectral line of the incoming pulses and is obtained by employing a line-by-line modulation scheme [6]. Some proposals addressing this objective have been made based on diverse high-resolution filtering techniques, such as microring resonators [7] or volume optics [8].

In this Letter, a scheme for an SPE-OCDMA system based on the use of the temporal self-imaging effect on fiber [9] is presented. As in other dispersion-based systems $[4,5]$, incorporating the codes with an electro-optic modulator enables their rapid reconfiguration. However, unlike with other techniques, the use of the self-imaging effect allows the individual line-by-line coding of the transmitted signal, making possible the simultaneous transmission of WDM and OCDMA signals within the same optical fiber. Furthermore, the speed at which the code is imposed using the modulator is the same as the data rate, avoiding the use of high-bandwidth modulators.

The proposed setup for the network can be seen in Fig. 1. A star coupler is used to interconnect the different users, where an optical circulator separates the transmitted signal (upper branch) from the received signal (lower branch). To clarify the working principle of the coder, a schematic illustration of the signals and their time-frequency representations at different points of the transmitter (labeled from A to D in Fig. 1.) is presented in Fig. 2.

A pulsed source is used to generate a train of ultrashort optical pulses at a fixed repetition rate $T_{0}$. Data are modulated at this rate to the train in an on-off keying scheme with an intensity modulator, obtaining at its output the train of pulses shown in Fig. 2(A). The resulting signal is passed through a linearly chirped fiber Bragg

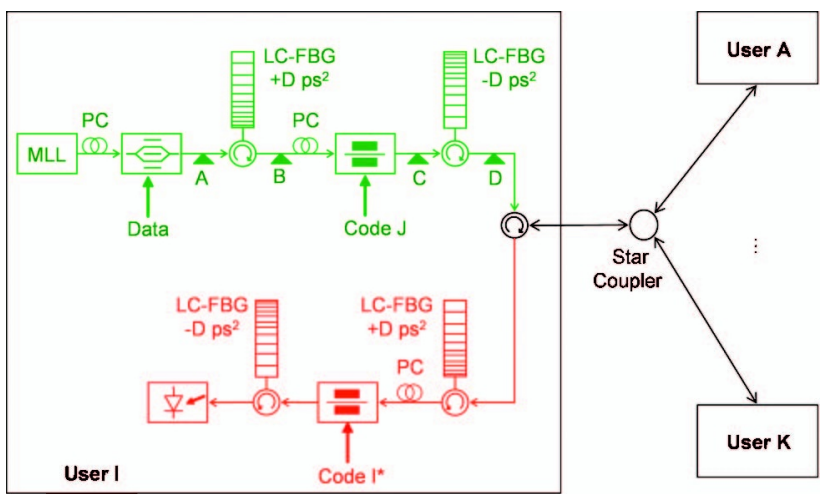

Fig. 1. OCDMA network scheme for the proposed setup with $K$ users. The upper branch of user $I$ (green) corresponds to the transmitter and the lower branch (red) to the receiver. Code $J$ is used at the transmitter to communicate with user $J$, while code $I^{*}$ is used at the receiver to decode the information (MLL, modelocked laser; PC, polarization controller; LC-FBG, linearly chirped fiber Bragg grating). 
grating (LC-FBG) that filters the signal to reduce the spectrum to only $N$ spectral lines, $N$ being the number of chips of the code. The LC-FBG also applies a chromatic dispersion $\left[D=T_{0}^{2} /(2 \pi)\right]$ that was selected to achieve a first-order temporal self-imaging effect [9]. As a result, the output signal is another train of pulses with the same repetition rate as the original, $T_{0}$, and with a temporal broadening in the individual pulses due to the filtering applied, as depicted in Fig. 2(B). The spectral components of each of the input pulses to the LC-FBG (represented with the same color in Fig. 2) experience different delays owing to the chromatic dispersion introduced by the grating. The value of that dispersion is chosen so that each of those spectral lines appears at the LC-FBG output in a different bit. Hence, as seen in Fig. 2(B), in each bit at the LC-FBG output, a new pulse is formed by combining spectral components from different pulses of the input (different colors in Fig. 2). For the coding, these new pulses are phase modulated by an electro-optic modulator so that a chip of the code word is introduced in each bit. This means that the rate at which the modulator is driven is the same as the data rate, $T_{0}$. However, the spectral lines coming from the same input pulse (same color in the figure) are coded with different phase shifts, that is, different chips, since they are placed in different bits [see Fig. 2(C)]. Once the phase shifts are introduced, a new temporal reordering is produced by a second LC-FBG with a dispersion chosen in order to make all the spectral lines of the same pulse at the coder input (same color) to coincide again in the same bit, as represented in Fig. 2(D). As a result of this, each spectral line of one of the output pulses acquires a different phase shift [Fig. 2(D)], resulting in an SPEOCDMA signal that is transmitted to the network.

To decode the signal, the procedure to be followed is analogous to the one used in the coder but applying the complementary code, as can be seen in the lower branch of Fig. 1. If an incorrect code is used, the phases shifts applied to the signal are not removed, resulting in a noisy signal spread along the bit period at the output of the decoder. This signal is recognized as an interfering noise, and it can be removed by introducing an optical time gate $[\underline{8}, 10]$. However, when the correct code is used, the phase shifts are removed, producing the recompression of the pulse at the center of the bit period and enabling the recovery of the information transmitted.

To experimentally verify the proposed configuration, the system was set up in the laboratory. The source used was a Calmar mode-locked laser with a frequency repetition rate of $10 \mathrm{GHz}$ and a pulse width of approximately

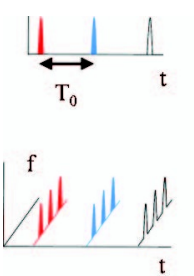

A
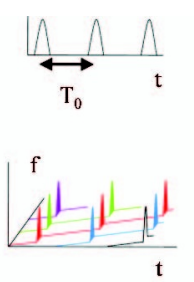

B

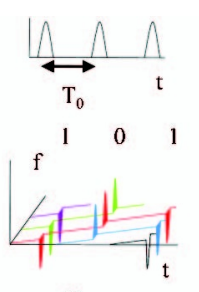

C
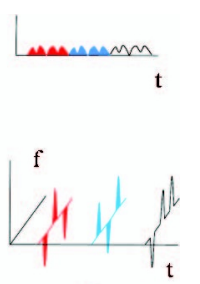

$\mathrm{D}$
Fig. 2. Representation of the signals and their corresponding time-frequency diagrams at the different steps of the coder as labeled in Fig. 1 .
5 ps. For simplicity, no data were modulated to the train of pulses. Figure 3 shows the power spectrum and eye diagram for the attenuated output of the laser. The amplitude responses of the LC-FBGs used in the setup had an FWHM bandwidth of $0.7 \mathrm{~nm}$, which limited the number of spectral lines to eight and therefore the length of the codes to $N=8$. To obtain the required reordering of the spectral components, the dispersion slope of the gratings was $\pm D= \pm 1590 \mathrm{ps}^{2}$. The codes used were a subset of the Hadamard family and were imposed using an electro-optic phase modulator driven by a code generator at 10 Gbps. To verify the correct decoding of the pulses, a back-to-back configuration was used, analyzing the resulting signals with a photodiode and sampling scope with a $65 \mathrm{GHz}$ bandwidth. An erbium-doped fiber amplifier was used between the coder and the decoder to partially compensate the losses of the system.

The measured signals at the output of the coder are shown in Fig. 4. As it can be observed, the spectrum of the coded signal [Fig. 4(a)] is band limited to eight spectral lines by the filtering applied, which corresponds to a bandwidth of approximately $0.7 \mathrm{~nm}$. The eye diagrams resulting from the application of codes 10101100, 10101010, and 11010010 are presented in Figs. 4(b)-4(d). As expected, they no longer correspond to a train of pulses but to a noisy signal spread along the bit period, depending on the specific code applied in each case. Figure $\underline{5}$ shows the eye diagrams at the output of the system when the signal has been coded using the code 10101010 [Fig. 4(c)]. As can be seen, only when the complementary code is used [Fig. 5(a)] is the signal correctly decoded, producing a recompression of the pulses transmitted at the center of the bit period and allowing its correct detection. Because of the encoding/decoding process, there is degradation in the detected signal when compared to the MLL output. Estimations based on the eye diagrams from Fig. 3(b) and Fig. 5(a) show a power penalty in the $Q$ factor of $6 \mathrm{~dB}$. On the other hand, when an incorrect code is used [Figs. 5(b)-5(d)], the decoded signal is spread along the bit period, rendering the recovery of transmitted information impossible. If more than one user is transmitting simultaneously, the detected signal is the combination of the signals, degrading further the quality of the received eye. This interference, usually known as multiuser access interference, is the main cause of system performance limitation in all OCDMA systems. However, in our case, as can be observed from the figures, most of the interfering signal is in the sides of the bit period, making recovery of the information possible by means of techniques for its

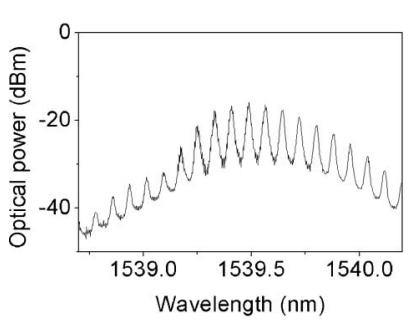

(a)

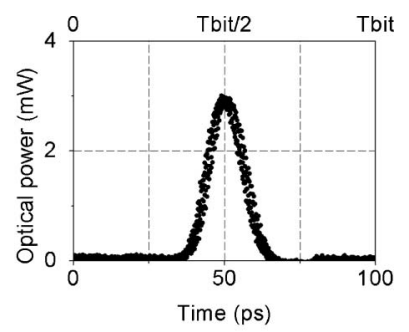

(b)
Fig. 3. Attenuated signal at the MLL output: (a) power spectrum, (b) eye diagrams. 


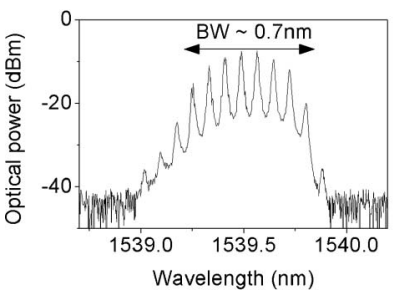

(a)

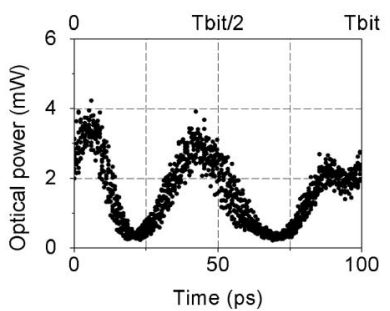

(c)

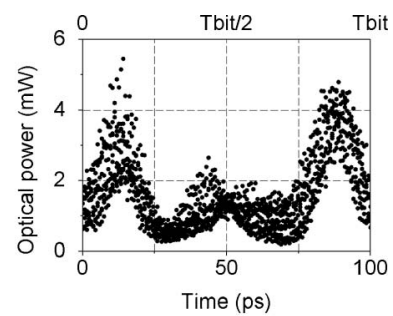

(b)

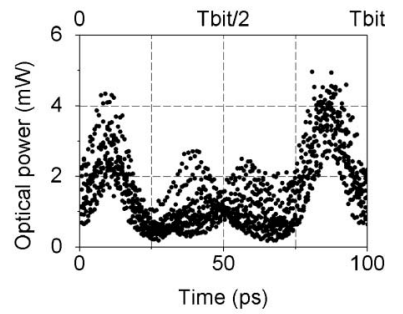

(d)
Fig. 4. Transmitted signal: (a) power spectrum, and eye diagrams when using the codes (b) 10101100, (c) 10101010, and (d) 11010010 .

removal that have already been proven in other schemes, such as optical time gating or optical thresholding $[8,10]$.

To summarize, an SPE-OCDMA coder/decoder based on the temporal self-imaging effect has been proposed. A proof of concept has been given by the experimental transmission of an unmodulated train of pulses with a $10 \mathrm{GHz}$ repetition rate. The coding and decoding are introduced by an electro-optic phase modulator driven by a 10 Gbps code, chosen as a subset of the Hadamard codes with a length of eight chips. The use of this electro-optic element at the decoder requires a strict control of the polarization at its input so that the the signal is correctly phase modulated. Otherwise the quality of the received signal could be compromised, resulting in an increased bit-error rate. The system is completely integrated in fiber, avoiding problems inherent in other proposed schemes, such as temperature control or the requirement for high-bandwidth electro-optic modulators. The use of line-by-line modulation permits also the simultaneous transmission of WDM signals within the fiber, as the spectrum is band limited to $0.7 \mathrm{~nm}$.

The authors wish to acknowledge financial support from the Comisión Interministerial de Ciencia y Tecnología (CICYT) of Spain via projects TEC2007-68065-C03-0102-03 and TEC2010-21303-C04-01-02.

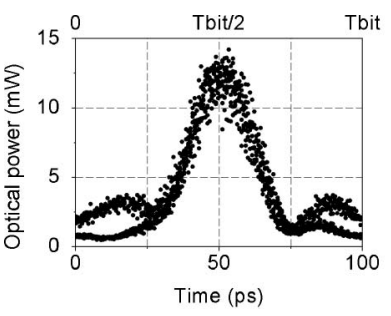

(a)

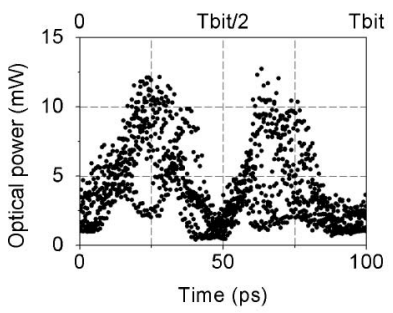

(c)

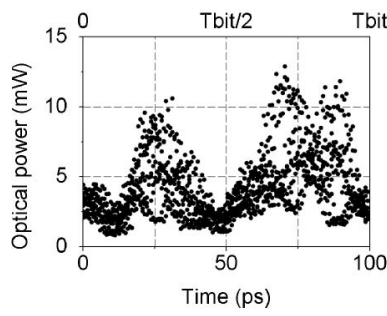

(b)

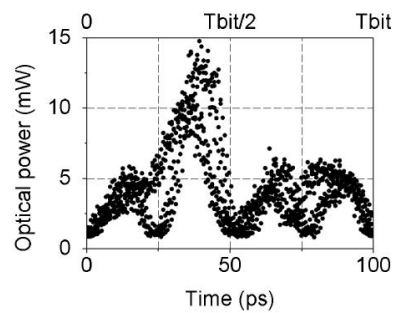

(d)
Fig. 5. Eye diagrams corresponding to the decoded signal when code 10101010 [Fig. 4(c)] is used at the encoder and codes (a) 01010101, (b) 01101001, (c) 00101101, and (d) 00110011 are used at the receiver.

\section{References}

1. P. R. Prucnal, ed., Optical Code Division Medium Access: Fundamentals and Applications (CRC Press, 2006).

2. J. A. Salehi, A. M. Weiner, and J. P. Heritage, J. Lightwave Technol. 8, 478 (1990).

3. R. P. Scott, V. J. Hernandez, N. K. Fontaine, F. M. Soares, R. Broeke, K. Perry, G. Nowak, C. Yang, W. Cong, K. Okamoto, B. H. Kolner, J. P. Heritage, and S. J. B. Yoo, IEEE J. Sel. Top. Quantum Electron. 13, 1455 (2007).

4. A. Andueza, D. Lasaosa, and D. Benito, Opt. Commun. 281, 5973 (2008).

5. X. Wang and N. Wada, Opt. Express 15, 7319 (2007).

6. Z. Jiang, C. B. Huang, D. E. Leaird, and A. M. Weiner, J. Opt. Soc. Am. B 24, 2124 (2007).

7. A. Agarwal, P. Toliver, R. Menendez, S. Etemad, J. Jackel, J. Young, T. Banwell, B. E. Little, S. T. Chu, W. Chen, J. Hryniewicz, F. Johnson, D. Gill, O. King, R. Davidson, K. Donovan, and P. J. Delfyett, J. Lightwave Technol. 24, 77 (2006).

8. S. Etemad, P. Toliver, R. Menendez, J. Young, T. Banwell, S. Galli, J. Jackel, P. Delfyett, C. Price, and T. Turpin, IEEE Photon. Technol. Lett. 17, 929 (2005).

9. J. Azaña and M. A. Muriel, IEEE J. Sel. Top. Quantum Electron. 7, 728 (2001).

10. W. Cong, C. Yang, R. P. Scott, V. J. Hernandez, N. K. Fontaine, B. H. Kolner, J. P. Heritage, and S. J. B. Yoo, IEEE Photon. Technol. Lett. 18, 1567 (2006). 